\title{
Mild cognitive impairment in novel SPG11 mutation-related sporadic hereditary spastic paraplegia with thin corpus callosum: case series
}

\author{
Chuan $\mathrm{Li}^{\dagger}$, Qi Yan ${ }^{\dagger}$, Feng-ju Duan ${ }^{\dagger}$, Chao Zhao ${ }^{\dagger}$, Zhuo Zhang ${ }^{\dagger}$, Ying Du* and Wei Zhang ${ }^{*}$ (D)
}

\begin{abstract}
Background: SPG11 mutation-related autosomal recessive hereditary spastic paraplegia with thin corpus callosum (HSP-TCC) is the most common cause in complicated forms of HSP, usually presenting comprehensive mental retardation on early-onset stage preceding spastic paraplegias in childhood. However, there are many instances of sporadic late-onset HSP-TCC cases with a negative family history, and potential mild cognitive deficits in multiple domains may be easily neglected and inaccurately described.

Methods: In this study, we performed next generation sequencing in four sporadic late-onset patients with HSPTCC, and combined Mini-Mental State Examination (MMSE) and Montreal Cognitive Assessment (MoCA) to evaluate cognition of the patients.

Results: By evolutionary conservation and structural modeling analysis, we have revealed 4 novel pathogenic SPG11 mutations, and firstly confirmed mild cognitive impairment (MCI) with normal MMSE scores $(\geq 27)$ and decreased MoCA scores $(<26)$ in these SPG11 mutation-related HSP-TCC patients, predominantly presenting impairment of executive function, delayed recall, abstraction and language.

Conclusions: The results expand the mutational spectrum of SPG11-associated HSP-TCC from sporadic cases, and confirm MCI with combination of decreased MoCA and normal MMSE assessment, suggesting that clinicians should consider doing a MoCA to detect MCl in patients with HSP, particularly those with HSP-TCC.
\end{abstract}

Keywords: SPG11, Next generation sequencing, Hereditary spastic paraplegia, Mild cognitive impairment

\section{Background}

Hereditary spastic paraplegia (HSP) is a heterogeneous group of genetically-driven neurodegenerative disorder, inherited in autosomal dominant (AD), autosomal recessive (AR) or X-linked patterns with onset age varying from infancy to late adulthood, and traditionally

\footnotetext{
* Correspondence: tdduying@fmmu.edu.cn; tdzw@fmmu.edu.cn

${ }^{\dagger}$ Chuan Li, Qi Yan, Feng-ju Duan, Chao Zhao and Zhuo Zhang contributed equally to this work.

Department of Neurology, Tangdu Hospital, Fourth Military Medical University, Xi'an City 710038, Shaanxi Province, China
}

classified into pure and complicated forms [1]. Among them, pure HSP is characterized by bladder involvement, weakness in isolation, slowly progressive spasticity and impaired vibration sense in the lower limbs, while complicated HSP is characterized by spastic paraplegias associated with additional neurological features such as cognitive deficits, thinning corpus callosum (TCC), seizures, amyotrophy, ataxia, extrapyramidal disturbance, visual or auditory disturbances, orthopaedic abnormalities and peripheral neuropathy [2]. Up to now, at least 84 different loci and 67 genes have been shown to be

(c) The Author(s). 2021 Open Access This article is licensed under a Creative Commons Attribution 4.0 International License, which permits use, sharing, adaptation, distribution and reproduction in any medium or format, as long as you give appropriate credit to the original author(s) and the source, provide a link to the Creative Commons licence, and indicate if changes were made. The images or other third party material in this article are included in the article's Creative Commons licence, unless indicated otherwise in a credit line to the material. If material is not included in the article's Creative Commons licence and your intended use is not permitted by statutory regulation or exceeds the permitted use, you will need to obtain permission directly from the copyright holder. To view a copy of this licence, visit http://creativecommons.org/licenses/by/4.0/ The Creative Commons Public Domain Dedication waiver (http://creativecommons.org/publicdomain/zero/1.0/) applies to the data made available in this article, unless otherwise stated in a credit line to the data. 
associated with HSP. Among these genes, SPG11 is the most common cause of complicated autosomal recessive hereditary spastic paraplegia with thin corpus callosum (HSP-TCC), which has been reported to be homozygous or compound heterozygous mutations [3].

General cognitive deficits in SPG11-related HSP-TCC preceding to spastic paraplegia is usually first noticed in childhood for learning difficulties and progresses insidiously to severe functional disability, diagnosed as mental retardation [4]. However, there are still lots of sporadic late-onset SPG11-related HSP-TCC cases without positive family history, and hardly diagnosed by only clinical manifestations and neuroimaging. Moreover, these patients may present spastic paraplegia without cognitive complaints, and potential mild cognitive deficits in multiple domains may be easily neglected and inaccurately described by conventional Mini-Mental State Examination (MMSE) assessment [5-7].

In this study, we performed a combined approach of next generation sequencing, evolutionary conservation and structural modeling analysis to genetically assess 4 sporadic HSP-TCC patients without positive family history and prominent cognitive impairment. The results revealed 1 reported and 4 novel pathogenic SPG11 mutations. Furthermore, we combined MMSE and Montreal Cognitive Assessment (MoCA) to evaluate cognition of the patients, and firstly confirmed mild cognitive impairment (MCI) with normal MMSE scores $(\geq 27)$ and decreased MoCA scores $(<26)$, predominantly presenting impairment of multiple cognitive domains including executive function, delayed recall, abstraction and language, which should be paid more attention by neurologists.

\section{Methods}

\section{Patients and clinical assessments}

Four cases with sporadic HSP were recruited. The clinical assessments were approved by the Expert Committee (equal to the Institutional Review Board) of the Tangdu Hospital of Fourth Military Medical University (China), and we have obtained the written informed consents from all the patients and their family members. The patients and their relatives were all Chinese.

Except positive family history and prominent cognitive impairment in complaints, the characteristics in our patients were consistent with the clinical and radiological criteria for the complicated form of HSP-TCC reported in Japan [8], and Italy [9]. The diagnosis was determined by at least three experienced neurologists and radiologists. Because of negative family history, all known possible causes of spastic paraplegia were carefully excluded. All the patients were assessed by Spastic Paraplegia Rating Scale (SPRS) for spastic paraplegia assessment [10], brain and spinal cord MRI scan, nerve conduction studies (NCS) and electromyography (EMG).

\section{Next generation sequencing}

The blood samples were collected for genetic analysis from 4 patients and all their relatives with informed consent. Extraction of genomic DNA from the peripheral blood leukocytes was obtained by using a standard protocol. Genomic DNA was isolated from peripheral leukocytes, fragmented into $150-200 \mathrm{bp}$ length with the use of sonication. The DNA fragments were then processed by end-repairing, A-tailing and adaptor ligation, a 4-cycle pre-capture PCR amplification, and enriched by a custom-designed panel capturing the coding exons of 39 genes associated with spastic paraplegia, including SPG11. Paired-end sequencing (150 bp) was performed on Illumina HiSeq X-ten platform to provide a mean sequence coverage of more than $100 \times$, with more than $95 \%$ of the target bases having at least $20 \times$ coverage.

Raw data was processed by the Illumina pipeline (version 1.3.4) for image analysis, error estimation, base calling and generating the primary sequence data. For the quality control, the Cutadapt (https://pypi.python.org/ pypi/cutadapt) and FastQC (www.bioinformatics. babraham.ac.uk/projects/fastqc/) were used to remove 3' $-/ 5^{\prime}$ - adapters and low quality reads, respectively. The clean reads were mapped to the human reference genome (UCSC hg19) with the use of the BWA (version 0.7.10, http://bio-bwa.sourceforge.net) [11], duplicate sequence reads were removed by Picard (version 1.85; http://picard.sourceforge.net), and GATK (version 3.1, https://software.broadinstitute.org/gatk/) [12], was used to detect variants. Variants were annotated by ANNOVAR software (version 2015Dec14, http://www. openbioinformatics.org/annovar/) [13], which including function implication (gene region, functional effect, mRNA GenBank accession number, amino acid change, cytoband, etc.) and allele frequency in dbSNP138, 1000 Genomes (Phase3 - Variant Frequencies 5b) and ExAc (exac.broadinstitute.org/) [14], referring to transcriptNM_025137. Damaging missense mutations were predicted by SIFT (sift.bii.a-star.edu.sg/) and PolyPhen-2 (genetics.bwh.harvard.edu/pph2/). Interpretation of the variants according to the American College of Medical Genetics and Genomics (ACMG) recommended standards [15], and all the variants will be categorized into pathogenic, likely pathogenic, uncertain significance (VUS), likely benign and benign.

Sanger sequencing was performed to validate the putative pathogenic variants, allowing segregation analyses where possible. Genetic information of healthy Chinese obtained from local Chinese Millionome Database (CMDB) was identified as healthy controls. 


\section{Structural and functional analysis}

SPG11 protein sequence was obtained from the uniprot database (https://www.uniprot.org/uniprot/Q96JI7). Conserved domain database (CDD) version 3.18 [16] was used to detect conserved structure domain in SPG11 via RPS-blast with position-specific score matries (PSSMs), Expect Value threshold was set to 0.01 .

Polyphen2 was used to predict possible structural or functional impact of amino acid substitution detected in human proteins using physical and evolutionary comparative algorithm, default setting was used. The prediction was against a precomputed database comprising 150 million missense SNPs detected in all exons of UCSC human genome (hg19) [17].

The orthologous genes of SPG11 were detected via blast, and the evolution tree was drawn by the gene orthology/paralogy predition method implemented in the ensemble database (http://asia.ensembl.org). Multiple sequence alignment was made using muscle 3.8 [18], Green bars shows areas of conserved peptides in the sequence, white areas are gaps in the alignment. The multiple alignment sequence used to draw the seqlogo figure using the weblogo software [19] (http://weblogo. berkeley.edu/). The sequence logo consists of stacks of symbols for corresponding amino acids. The height of the stack indicates the conservation of amino acids. Chemical properties of amino acids were used to define the color system: polar amino acids $(\mathrm{G}, \mathrm{S}, \mathrm{T}, \mathrm{Y}, \mathrm{C}, \mathrm{Q}, \mathrm{N})$ are green, basic $(K, R, H)$ blue, acidic $(D, E)$ red and hydrophobic (A, V, L, I, P, W, F, M) amino acids are black.

De novo 3D structure modeling was performed using the I-TASSER algorithm [20] for both wild and mutant SPG11, It identified first 10 possible structure templates using a meta-server threading approach LOMETS [21] based on the highest significance Z-score of the threading alignments, and used SPICKER program to select the final simulation model based on pair-wise structure similarity using RMSD (TM-score). The confidence of the model is quantitatively measured by $\mathrm{C}$-score. The first model with the best $\mathrm{C}$-score is selected for further analysis.

Simulated protein 3D structures of wild and mutant SPG11 were aligned using superpose version 1.0 [22]. Protein super positions were calculated using a quaternion approach. Rasmol version 2.7 [23] was used to visualize the wild and mutant structures. Relative position of mutation site was determined using structure alignments.

\section{Neuropsychological evaluation}

All the patients were assessed by Neuropsychiatric Inventory (NPI), MMSE and MoCA for neuropsychological assessments. The NPI and MMSE were administered at the beginning, followed by MoCA on 7th hospital day to avoid effects of habituation. A cutoff of $\geq 27$ on the MMSE was chosen to indicate normal cognitive function and the accepted cutoff of $<26$ on the MoCA was taken to indicate cognitive impairment [24, 25]. A cutoff of $\geq 26$ on the ADL was chosen to indicate functional disability.

MoCA is a 30-point test administered in 10 minconsisting of seven subtests. Executive functions are assessed using a clock-drawing task ( 3 points), a threedimensional cube copy (1point) and the Trail Making B task (1 point). Naming is assessed using a three-item confrontation naming task with low-familiarity animals (lion, camel, rhinoceros; 3 points). Attention is evaluated using a sustained attention task (target detection using tapping; 1 point), a serial subtraction task ( 3 points) and digits forward and backward (1 point each). Language is assessed using repetition of two syntactically complex sentences ( 2 points) and a phonemic fluency task (1 point). Abstraction is assessed using a two-item verbal abstraction task ( 2 points). The short-term memory recall task (5 points) involves two learning trials of five nouns and delayed recall after approximately $5 \mathrm{~min}$. Finally, orientation to time and place is evaluated (6 points) [25].

\section{Results}

\section{Clinical features}

Four sporadic spastic paraplegias patients (2 male and 2 female) and their close relatives were studied. Ages at onset were based on information provided by the patients, the median age at onset for motor symptoms was 23 years (ranging from 18 to 27 years), and the median course of disease was 4 years (ranging from 3 to 7 years). The symptoms at onset were all spasticity, and the spastic paraplegia was evaluated by Spastic Paraplegia Rating Scale (SPRS). Furthermore, no dysarthria, dysphagia, skeletal deformity, cerebellar signs, ophthalmoplegia, decreased vision, sphincter disturbance, amyotrophy, extrapyramidal signs, epilepsy, cataract, or optic atrophy was found in each patient. All patients showed normal in both NCS and EMG examinations. The clinical features of patients were listed in Table 1. Brain MRI showed thinning of whole corpus callosumin all patients, mostpronounced in the genu and body parts, relatively spared in the splenium, without obvious periventricular white matter changes or cortical atrophy, while whole spinal cord MRI of each patient was normal (Fig. 1a-c).

\section{Genetic findings}

In this study, we performed a custom-designed panel sequencing by next-generation sequencing in four sporadic patients with spastic paraplegia and TCC, and identified novel as well as reported SPG11 mutations. Five 
Table 1 Summary of clinical presentations and novel mutations in sporadic SPG11-related HSP patients in this study

\begin{tabular}{|c|c|c|c|c|c|}
\hline & Patient 1 & Patient 2 & Patient 3 & & Patient 4 \\
\hline Inheritance & Sporadic & Sporadic & Sporadic & & Sporadic \\
\hline Sex & $\mathrm{F}$ & $\mathrm{F}$ & M & & M \\
\hline Schooling years & 14 & 16 & 15 & & 14 \\
\hline Initial symptoms & Spasticity & Spasticity & Spasticity & & Spasticity \\
\hline UL reflexes & ++ & ++ & ++ & & ++ \\
\hline LL reflexes & ++++ & ++++ & +++ & & +++ \\
\hline SPRS & 9 & 10 & 7 & & 7 \\
\hline NPI & 0 & 0 & 0 & & 0 \\
\hline MMSE & $30 / 30$ & $30 / 30$ & $29 / 30$ & & $28 / 30$ \\
\hline MoCA & $22 / 30$ & $18 / 30$ & $16 / 30$ & & $17 / 30$ \\
\hline $\begin{array}{l}\text { Executive } \\
\text { function }\end{array}$ & $2 / 5$ & $1 / 5$ & $1 / 5$ & & $2 / 5$ \\
\hline Delayed recall & $2 / 5$ & $2 / 5$ & $1 / 5$ & & $1 / 5$ \\
\hline Abstraction & $1 / 2$ & $0 / 2$ & $0 / 2$ & & $0 / 2$ \\
\hline Language & $2 / 3$ & $1 / 3$ & $0 / 3$ & & $0 / 3$ \\
\hline Naming & $3 / 3$ & $3 / 3$ & $3 / 3$ & & $3 / 3$ \\
\hline Attention & $6 / 6$ & $5 / 6$ & $5 / 6$ & & $6 / 6$ \\
\hline Orientation & $6 / 6$ & $6 / 6$ & $6 / 6$ & & $5 / 6$ \\
\hline \multicolumn{6}{|l|}{ MRI } \\
\hline TCC & + & + & + & & + \\
\hline PWM changes & - & - & - & & - \\
\hline $\begin{array}{l}\text { Cortical } \\
\text { atrophy }\end{array}$ & - & - & - & & - \\
\hline $\begin{array}{l}\text { Ventricular } \\
\text { Dilation }\end{array}$ & - & - & - & & - \\
\hline $\begin{array}{l}\text { Cerebellar } \\
\text { atrophy }\end{array}$ & - & - & - & & - \\
\hline Spinal cord & - & - & - & & - \\
\hline \multicolumn{6}{|l|}{ Novel mutations } \\
\hline Inheritance & Homozygous & Homozygous & Compound heterozygous & & Homozygous \\
\hline Location & exon 28 & exon 2 & exon 30 & intro 31 & exon 30 \\
\hline Mutation & $\begin{array}{l}\text { c. } 4834 C>T \\
\text { chr15:44881522 }\end{array}$ & $\begin{array}{l}\text { c.316G >C } \\
\text { chr15:44952756 }\end{array}$ & $\begin{array}{l}\text { c. } 5609 \mathrm{~T}>\mathrm{A} \\
\text { chr15:44876741 }\end{array}$ & $\begin{array}{l}\text { c.5867-1G > T } \\
\text { chr15:44867240 }\end{array}$ & $\begin{array}{l}\text { c.5137C > T } \\
\text { chr15:44876741 }\end{array}$ \\
\hline Consequence & Stop codon & $\begin{array}{l}\text { Missense } \\
\text { mutation }\end{array}$ & Stop codon & Frameshift mutation & Stop codon \\
\hline Effect & p.Q1612X & p.A106P & p.L1870X & & p.Q1713X \\
\hline ACMG criteria & $\begin{array}{l}\text { PVS1 + PM2 + PM3 } \\
\text { (pathogenic) }\end{array}$ & $\begin{array}{l}\text { PM2 + PM3 } \\
\text { (VUS) }\end{array}$ & $\begin{array}{l}\text { PVS1 + PM2 + PM3 } \\
\text { (pathogenic) }\end{array}$ & $\begin{array}{l}\text { PVS1 + PM2 + PM3 } \\
\text { (pathogenic) }\end{array}$ & $\begin{array}{l}\text { PVS1 + PM2 + PM3 } \\
\text { (pathogenic) }\end{array}$ \\
\hline
\end{tabular}

M Male, F Female, UL Upper limbs, LL Lower limbs, SPRS Spastic Paraplegia Rating Scale, NPI Neuropsychiatric Inventory, MMSE Mini Mental State Examination, MoCA Montreal Cognitive Assessment, nd Not done, MRI Magnetic resonance imaging, TCC Thin corpus callosum, PWM Periventricular white matter, + Presence;Absence, ACMG The American College of Medical Genetics and Genomics recommended standards, VUS Variants of uncertain significance

mutations were recognized, and fourof them, including 3 stop codon (c.4834C $>\mathrm{T}$ chr15:44881522/p.Q1612X, c.5137C > T chr15:44876741/p.Q1713X, c.5609 T > A chr15:44876741/p.L1870X) and 1 missense mutation (c.316G > C chr15:44952756/p.A106P), were identified for the first time. (Table 1). The mutations were homozygous in 3 patients, and compound heterozygous in 1 patient. Results of Sanger sequencing indicated that these mutations segregated with the disease in patients while healthy parents and siblings of patients were all asymptomatic heterozygous carriers (Fig. 1d-g). Copy number variants (CNVs) of genes listed in the panel were also tested, and no associated CNVs were identified. 


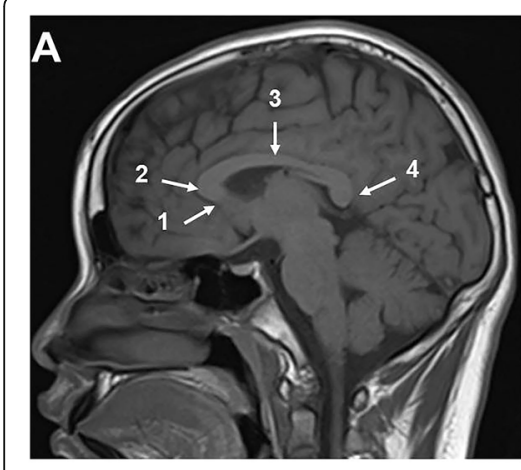

D

Patient 1: c.4834C>T chr15:44881522, p.Q1612X
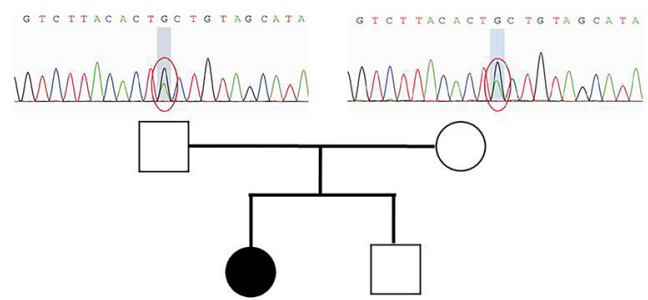

Exon 28: $+m \quad m m$

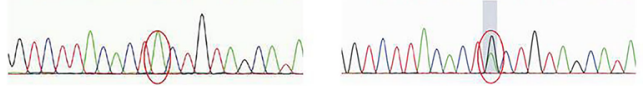

$\mathbf{F}$

Patient 3: c.5609T>A chr15:44876741, p.L1870X

c. 5867-1G > T chr15:44867240

c.5609T $>$ A chr15:44876741, p.L1870X

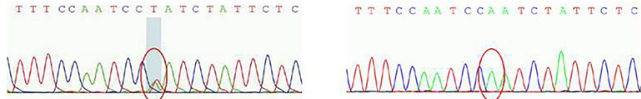
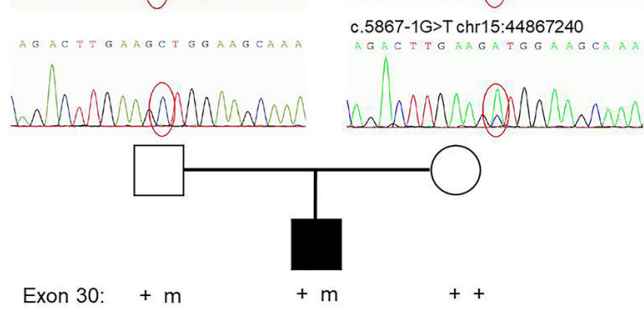

Intro 31: ++

$m+$

$m+$

c.5609T>A chr15:44876741, p.L1870X c.5867-1G>T chr15:44867240

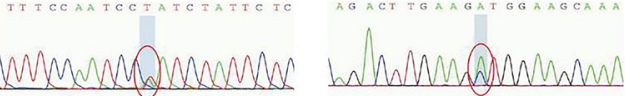
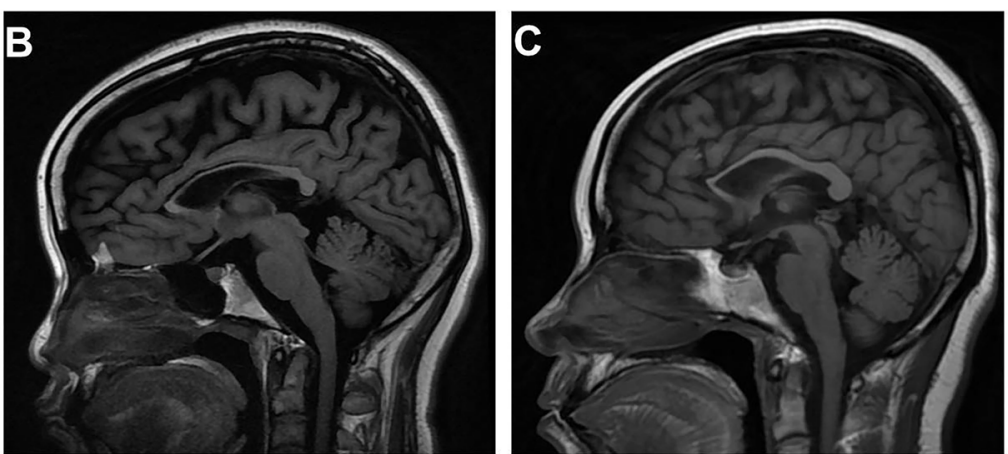

E

Patient 2: c.316G $>$ C chr15:44952756, p.A106P

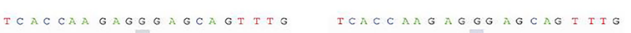

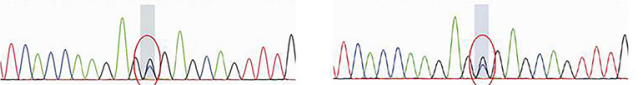

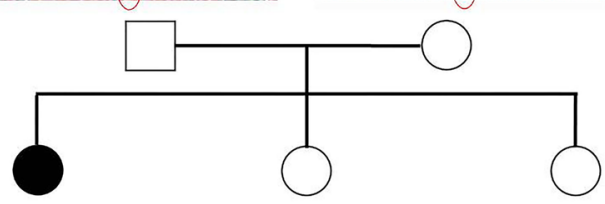

Exon 2: $\mathrm{mm}+\mathrm{m}+\mathrm{m} \quad+\mathrm{m} \quad+\mathrm{m}$

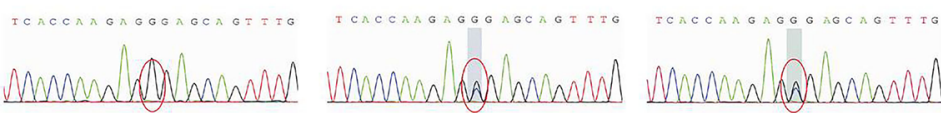

G

Patient 4: c.5137C>T chr15:44876741, p.Q1713X
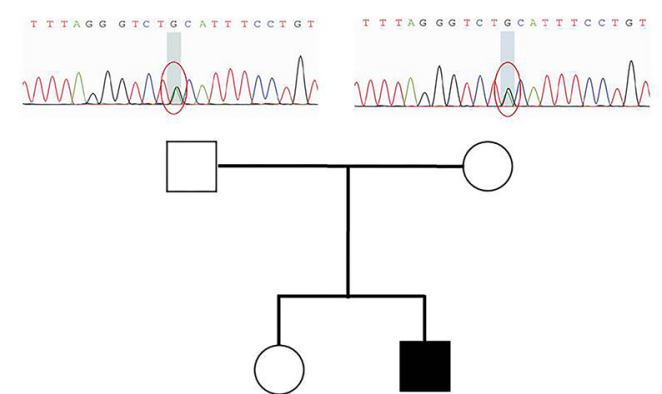

Exon 30: $\quad+m+m$

$m m+m$

TTIAGGGTCTGCA TT TCCTC

TTTAGG GICTACATTTCOTGT

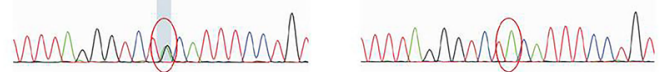

Fig. 1 Presentations of corpus callosum in normal and SPG11-related HSP-TCC patients in midline sagittal brain MRI. a T1-weighted MR image shows normal corpus callosum anatomy in a 24-year-old female (1: rostrum, 2: genu, 3: body, 4: splenium). T1-weighted MR images of patient 1 (b) and patient 2 (c) with SPG11 homozygous mutation show thinning of whole corpus callosum most pronounced in the genu and body parts, relatively spared in the splenium. Pedigrees and mutation segregation of the families with SPG11 mutations: patient 1 (d), patient 2 (e), patient 3 $(\mathbf{f})$, and patient $4(\mathbf{g})$. The squares indicate male; circles indicate female; filled shape, affected. M: mutation; +: wild type respectively. Chromatograms showing the mutations and respective wild type sequences are shown below the pedigrees

From patient 1, a novel homozygous stop coding mutation was detected in exon28 (c.4834C > T) of SPG11, predicted to truncate the functional protein (PVS1). This mutation was never reported in public genomic databases (ExAC or gnomAD), nor detected in healthy controls (PM2). According to ACMG criteria, this mutation was classified to be likely pathogenic (PVS1 + PM2).

The SPG11 gene of patient 2 displayed a novel homozygous missense c.316G >C (p.A106P) in exon 2. This missense was not identified in ExAC, gnomAD, or 
healthy controls (PM2). Some in silico algorithms, including SIFT and PolyPhen_2, performed the prediction of pathogenicity of this mutation. This mutation was graded as VUS based on the standard of ACMG. However, considering the absence of other variation or $\mathrm{CNV}$ in HSP related gene of this HSP-TCC patient, it is highly possible that this missense was the associated diseasecausing loci.

The sequencing result indicated heterozygous mutations that c.5609 T > A (p.L1870X) and c.5867-1G > T presented in two alleles of the SPG11 gene respectively in patient 3. c. $5609 \mathrm{~T}>\mathrm{A}$ was predicted to prematurely truncate the protein product (PVS1) and was neither reported in public genomic databases nor detected in healthy controls (PM2). It's in trans mutation, mutation c.5867-1G > T, was previously published as Pathogenic (rs1060501168) (PM3) [26]. Thus mutationc.5609 T > A was classified as Pathogenic (PVS1 + PM2 + PM3) according to ACMG criteria.

In patient 4, the novel homozygous nonsense was predicted to truncate the SPG11 in exon $30($ c. $5137 \mathrm{C}>\mathrm{T}$ ) (PVS1). This mutation was never reported in ExAC or gnom AD and was not detected in controls (PM2). Two patients were identified in this family with genotypes cosegregated with the disease (PP1). Therefore, this mutation was classified as pathogenic (PVS1 + PM2 + PP1) through ACMG standards.

To accept the variants found via NGS, we have preformed the sanger sequencing test. All 4 mutations were validated and confirmed.

\section{Pathogenicity of the mutations}

Conservation of these 4 mutations were calculated based on multiple sequence alignment of 141 SPG11 homologous sequences. All these 4 residues were evolutionarly conserved in over $50 \%$ orthologous. In patient 1 , the mutation site (Q1612X) was located at the 2001amino acid position where the main variants were $\mathrm{Q}$ and $\mathrm{R}$. Q is the most conserved, suggesting the importance of $\mathrm{Q}$. The stop gain mutation at this site may lead to the pathogenicity. In patient 2 , the mutation site (A106P) was located at the 167 amino acid position in the below seqlogo, where $\mathrm{A}, \mathrm{T}$ and $\mathrm{V}$ were common residues occurred in evolution across variants species. Alanine was the most conserved residue, however, Polin was rare. The Proline mutation was not presented in the evolution. In patient 3, the mutation site (L1870X) was located at the 2124 amino acid position where the main mutations was $\mathrm{L}$, suggesting the importance of $\mathrm{L}$ at this position. The stop gain mutation may lead to the pathogenicity. In patient 4, the mutation site (Q1713X) was located at the 2124 amino acid position and the main variants were $\mathrm{Q}$ and $\mathrm{N}$, and $\mathrm{Q}$ was the most conserved.
The stop gain mutation may lead to the pathogenicity. (Fig. 2a-d).

Among them, A106P in patient 2 was predicted to be probably damaging with polyphen-2 score 0.98 (sensitivity 0.76 , specificity 0.96 ), and the other 3 mutations in patient 1, 3 and 4 were all stop gains. To better understanding the role of these variants, structural modeling analysis was performed. The prediction with the Bepipred Linear Epitope Prediction 2.0 (http://www.cbs. dtu.dk/services/BepiPred/cite.php) showed that the mutation of A106P caused a sufficient surface exposure and orientation change of L104, and made the nearby peptide SRNSSTPTEKPKL $(92-104)$ to be a potential epitope, The other 3 variants were all stop gain mutations, causing the lost of the helix richc-terminal part of the SPG11, which could be important for structural stabilization.(Fig. 2e-h). Taking together, it seems that the cterminal domain of SPG11 could be an important binding domain with other proteins and it is stabilized with a helix rich structure to the core region of SPG11. All these 3 variants (Q1612X, Q1713X, L1870X) introduced stop codon to the beginning position of the binding domain, thereby causing damages to both the SPG11 core structure and potential protein interaction function.

\section{Neuropsychological findings}

All the patients have been educated for more than 12 years. NPI was performed in them for psychiatric assessment, and the results showed normal. MMSE was performed in these patients for preliminary cognitive assessment, and the score of each patient was normal ranging from 28 to 30 , according to accepted normal MMSE cutoff value $\geq 27$, suggesting no general cognitive decline or dementia. Moreover, all the patients were reevaluated by MoCA in detail, and the score of each patient was decreased ranging from 16 to 22 , according to accepted abnormal cutoff value $<26$. Among the seven subtests of MoCA, the average score of "Executive functions (5 points)" was 1.5 (ranging from 1 to 2 ); the average score of "Delayed recall (5 points)" was 1.5 (ranging from 1 to 2); the average score of "Abstraction (2 points)" was 0,25 (ranging from 0 to 1 ); the average score of "Language ( 3 points)" was 1 (ranging from 0 to $2)$; the average score of "Naming (3 points)" was 3; the average score of "Attention (6 points)" was 5.5 (ranging from 5 to 6); the average score of "Orientation (6 points)" was 5.75 (ranging from 5 to 6 ) (Table 1). The results of MoCA showed potential impairments in multiple cognitive domains that are not detected by the MMSE, including executive function, delayed recall, abstraction and language. Altogether, combination of MMSE and MoCA confirmed mild cognitive impairment (MCI) in these sporadic SPG11-related HSP-TCC cases. 


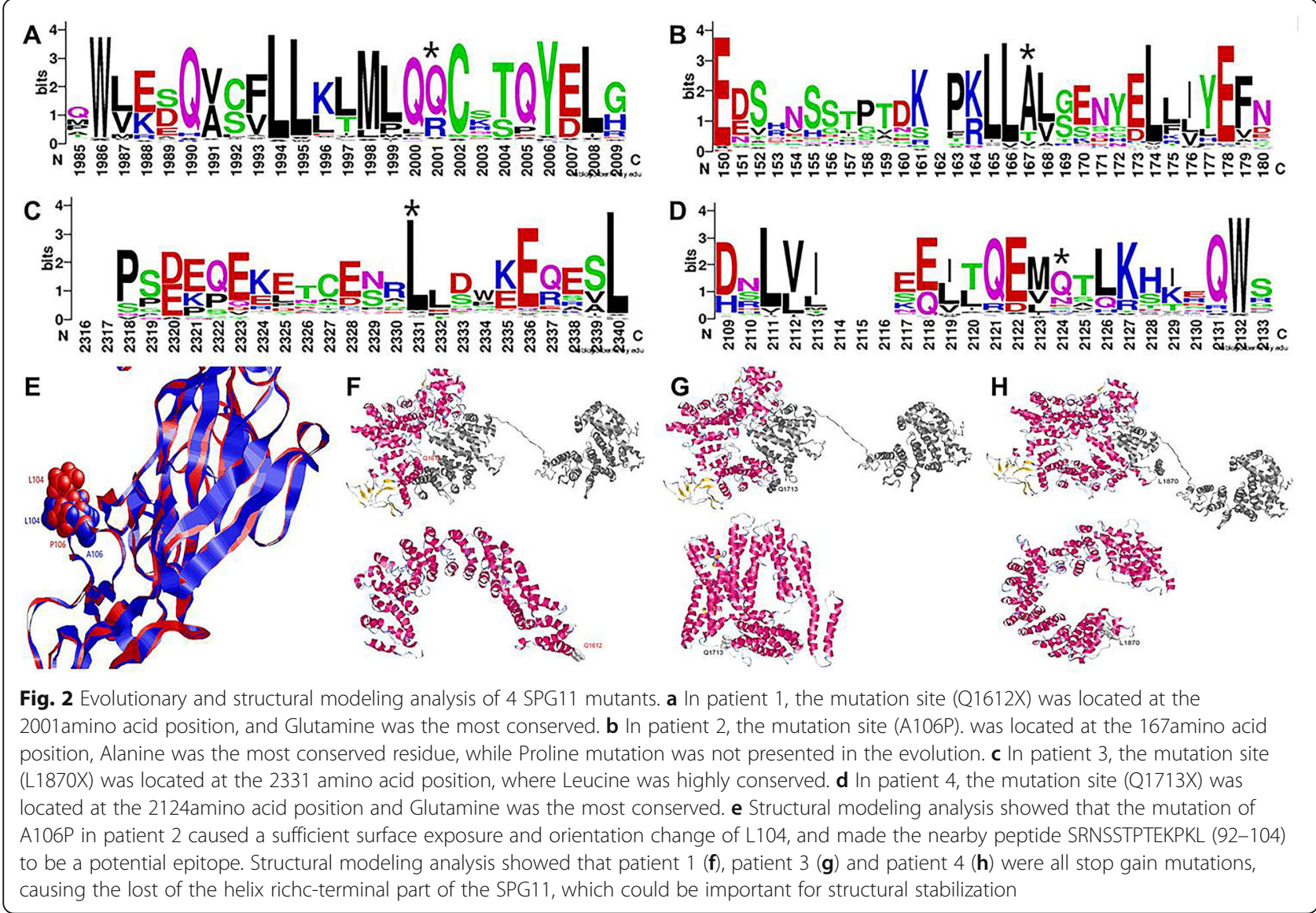

\section{Discussion}

In present study, we have reported four sporadic HSPTCC cases, and found novel SPG11 mutations in each patient by next generation sequencing. Among them, truncating variants included two homozygous stop-gain mutations in patient 1 and 4, and one compound heterozygous stop-gain and frameshift mutation in patient 3, while homozygous missense mutation in patient 4 belonged to non-truncating variant. Moreover, systematical evaluation showed that all 4 variants occur at evolutionary conserved residues, and present highly possible damages for either structural stabilization or potential protein binding ability of SPG11, suggesting pathogenenicity of these mutations. SPG11 gene encodes endogenous expression of spatacsin, distributed in the neurons forming the corticospinal tract and corpus callosum, as well as in hippocampus, cerebellum and dentate nucleus in adult CNS [27]. Loss of function may affect axonal transport and autolysosomes accumulation, contributing to diffuse neurodegeneration and causing clinical heterogeneity (presenting ataxia, parkinsonism or cognitive impairment in addition to core feature of spastic gait) in SPG11-related HSP-TCC [28, 29]. Among these complicated symptoms, non-movement disorders, such as cognitive profile and subtle deficits, may be easily ignored or inaccurately described. In previous retrospective studies about SPG11-related HSP-TCC, only $19 \%(4 / 21)$ patients has been reported cognitive impairments at onset, and almost $27 \%(4 / 15)$ patients has described as "TCC without cognitive impairment". After longitudinal follow-up, cognitive dysfunction clearly worsened with disease progression $[27,30]$.

The corpus callosum is the main commissural pathway linking the hemispheres of the brain, and identifiable anatomically divided into four parts: rostrum, genu anteriorly, body centrally and splenium posteriorly. Thinning of the corpus callosum (TCC) is a key manifestation and often only imaging feature in early SPG11-related familial or sporadic HSP, and almostly can be found in each patient, predominantly thinning in the genu and body, relatively sparing in the splenium of corpus callosum, which is also consistent with four sporadic patients reported in our study. The project fibers arising from prefrontal and motor cortex mainly pass through the genu and body respectively, and further research has also demonstrated that the prefrontal and motor portions of the corpus callosum are preferentially affected in SPG11-related HSP-TCC [31-33]. Moreover, voxel-based analysis in neuroimaging also showed global reduction of generalized fractional anisotropy (GFA) in 
the cerebral white matter innervating cortical regions, including prefrontal, parietal and temporal cortex, suggesting impaired microstructural integrity in fibers and restricted cerebral hypometabolism for diffuse cognitive deficits in SPG11-related HSP-TCC [31-33].

Although early and widespread impairment of cognitive functions across all domains is a well-known phenomenon in SPG11-related HSP-TCC, there are still some cases of TCC without cognitive impairment as reported previously. Potential mild cognitive deficits in some specific domains may be easily neglected by neurologist's clinical impression and inaccurately described by conventional MMSE, which is widely used for screening general cognitive decline or dementia, but inadequate for subtle cognitive impairment [5-7]. Unlike MMSE, MoCA is designed to adding assessments or increasing difficulties of tasks such as execution, abstraction, delayed recall and language, and widely used to evaluate mild cognitive impairment (MCI) in degenerative diseases [25]. More recently, a research about cognitive profiles in SPG4-related pure HSP has found that subtle cognitive deficits not compatible with dementia were detected by discrepancy between normal MMSE and decreased MoCA scores in adult patients, and there was similar cognitive performances between truncating (nonsense and frameshift mutations) and non-truncating (missense and in-frame insertion) variants, which is consistent with SPG11-related HSP-TCC patients reported in our study [25].

$\mathrm{MCI}$ is an intermediate clinical state between normal cognitive function and dementia with decline on objective cognitive tasks, and the aetiology of $\mathrm{MCI}$ is heterogeneous including different neurodegenerative diseases, ischaemia, trauma, metabolic disturbance, etc. [32]. Neuropsychological testing can be helpful to distinguish MCI from normal or dementia cases, for dementia usually presenting clear impairment in functional activities and scores low on MMSE, while MCI showing particularly subtle deficits by more sensitive measures such as MoCA [34]. In fact, $75 \%$ of patients with MCI on neuropsychological testing had normal MMSE but abnormal MoCA, and sensitivity of MoCA for MCI is almost 90$100 \%$ [25]. In our study, the combination of MMSE and MoCA has confirmed MCI in SPG11-related HSP-TCC, characteristically presenting potential deficits in multiple cognitive domains that were not detected by the MMSE, including executive function and abstraction (not tested by the MMSE) and delayed recall and language repetition (MMSE items too easy). These specific phenotypes of cognitive deficits in our patients were also in agreement with SPG4-related pure HSP as described before, suggesting that MoCA is likely to be a more sensitive screening tool for subtle cognitive changes in both pure and complicated HSP.

\section{Conclusions}

Collectively, in present study, we have performed next generation sequencing in four sporadic HSP-TCC patients without cognitive complaints, and revealed 3 homozygous and 1 compound heterozygous novel SPG11 mutations. In addition, we systematically evaluated the pathogenenicity of these mutations via evolutionary conservation and structural modeling analysis. We found that all 4 variants occur at evolutionary conserved residues, and the mutations were all highly possible damaging for either structural stabilization or potential protein binding ability of SPG11. Our findings expand the mutational spectrum of SPG11-associated HSP-TCC from sporadic cases. Moreover, we firstly confirm MCI by combination of MMSE and MoCA in these patients, characteristically presenting potential deficits in multiple cognitive domains (including executive function, delayed recall, abstraction and language) in SPG11related HSP-TCC, which should be paid more attention by neurologists.

\section{Abbreviations}

MCl: Mild cognitive impairment; HSP: Hereditary spastic paraplegias;

AD: Autosomal dominant; AR: Autosomal recessive; MRI: Magnetic resonance imaging; TCC: Thincorpus callosum; MMSE: Mini-Mental State Examination; MoCA: Montreal Cognitive Assessment; SPRS: Spastic ParaplegiaRating Scale; NPI: Neuropsychiatric Inventory; CMDB: Chinese Millionome Database; ACMG: American College of Medical Genetics and Genomics; VUS: Variant of Uncertain Significance; CNVs: Copy Number Variations

\section{Acknowledgements}

We thank the patients and relatives for their collaboration.

\section{Authors' contributions}

Conception and design of the study: WZ, YD. Acquisition of data: CL, QY, FD. Genetic analysis: QY, CZ, ZZ. Drafting of manuscript: CL, YD. Revision of the manuscript: WZ. All authors read and approved the final manuscript.

\section{Funding}

This work was supported by research grants from the National Natural Science Foundation of China $(81971003,81371209,81701041)$, Key project of research and development plan of Shaanxi Province (2018SF-086), Key project of basic natural science research program of Shaanxi Province (2019JZ-18), Tangdu hospital innovation development foundation (2019QYTS002, 2018QYTS010, 2016LYJ007). The funders had no role in study design, data collection and analysis, decision to publish or preparation of the manuscript.

\section{Availability of data and materials \\ The dataset analysed are available from the corresponding author on reasonable request.}

\section{Ethics approval and consent to participate}

This study has been approved by the Ethical Committee of Tangdu Hospital, Fourth Military Medical University. We have obtained the written informed consents from all the patients and their family members.

\section{Consent for publication}

We obtained written informed consent from each participant for publication of this report and any accompanying images. A copy of the written consent is available for review by the Editor-in-Chief of this journal.

Competing interests

The authors declare that they have no competing interests. 
Received: 17 August 2020 Accepted: 29 December 2020

Published online: 11 January 2021

\section{References}

1. Faber I, Pereira ER, Martinez ARM, Franca M Jr, Teive HAG. Hereditary spastic paraplegia from 1880 to 2017: an historical review. Arq Neuropsiquiatr. 2017:75(11):813-8

2. Kara E, Tucci A, Manzoni C, Lynch DS, Elpidorou M, Bettencourt C, et al. Genetic and phenotypic characterization of complex hereditary spastic paraplegia. Brain. 2016;139(Pt 7):1904-18.

3. Tesson C, Koht J, Stevanin G. Delving into the complexity of hereditary spastic paraplegias: how unexpected phenotypes and inheritance modes are revolutionizing their nosology. Hum Genet. 2015;134(6):511-38.

4. Ueda M, Katayama Y, Kamiya T, Mishina M, Igarashi H, Okubo S, et al. Hereditary spastic paraplegia with a thin corpus callosum and thalamic involvement in Japan. Neurology. 1998;51(6):1751-4.

5. Yoon WT, Lee WY, Lee ST, Ahn JY, Ki CS, Cho JW. Atypical hereditary spastic paraplegia with thin corpus callosum in a Korean patient with a novel SPG11 mutation. Eur J Neurol. 2012;19(1):e7-8.

6. Romagnolo A, Masera S, Mattioda A, Superti G, Santorelli FM, Mongini T, et al. Atypical hereditary spastic paraplegia mimicking multiple sclerosis associated with a novel SPG11 mutation. Eur J Neurol. 2014;21(2):e14-5.

7. Rubegni A, Storti E, Tessa A, Federico A, Santorelli FM. Hereditary spastic paraplegia type 11 with a very late onset. J Neurol. 2015;262(8):1987-9.

8. Shibasaki Y, Tanaka H, Iwabuchi K, Kawasaki S, Kondo H, Uekawa K, et al. Linkage of autosomal recessive hereditary spastic paraplegia with mental impairment and thin corpus callosum to chromosome 15A13-15. Ann Neurol. 2000;48(1):108-12.

9. Casali C, Valente EM, Bertini E, Montagna G, Criscuolo C, De Michele G, et al. Clinical and genetic studies in hereditary spastic paraplegia with thin corpus callosum. Neurology. 2004;62(2):262-8.

10. Schule R, Holland-Letz T, Klimpe S, Kassubek J, Klopstock T, Mall V, et al. The spastic paraplegia rating scale (SPRS): a reliable and valid measure of disease severity. Neurology. 2006;67(3):430-4.

11. Li H, Durbin R. Fast and accurate long-read alignment with burrowswheeler transform. Bioinformatics. 2010;26(5):589-95.

12. McKenna A, Hanna M, Banks E, Sivachenko A, Cibulskis K, Kernytsky A, et al. The genome analysis toolkit: a MapReduce framework for analyzing nextgeneration DNA sequencing data. Genome Res. 2010;20(9):1297-303.

13. Wang K, Li M, Hakonarson H. ANNOVAR: functional annotation of genetic variants from high-throughput sequencing data. Nucleic Acids Res. 2010; 38(16):e164.

14. Lek M, Karczewski KJ, Minikel EV, Samocha KE, Banks E, Fennell T, et al. Analysis of protein-coding genetic variation in 60,706 humans. Nature. 2016; 536(7616):285-91.

15. Richards S, Aziz N, Bale S, Bick D, Das S, Gastier-Foster J, et al. Standards and guidelines for the interpretation of sequence variants: a joint consensus recommendation of the American College of Medical Genetics and Genomics and the Association for Molecular Pathology. Genet Med. 2015; 17(5):405-24.

16. Lu S, Wang J, Chitsaz F, Derbyshire MK, Geer RC, Gonzales NR, et al. CDD/ SPARCLE: the conserved domain database in 2020. Nucleic Acids Res. 2020; 48(D1):D265-8.

17. Adzhubei IA, Schmidt S, Peshkin L, Ramensky VE, Gerasimova A, Bork P, et al. A method and server for predicting damaging missense mutations. Nat Methods. 2010;7(4):248-9.

18. Edgar RC. MUSCLE: multiple sequence alignment with high accuracy and high throughput. Nucleic Acids Res. 2004;32(5):1792-7.

19. Crooks GE, Hon G, Chandonia JM, Brenner SE. WebLogo: a sequence logo generator. Genome Res. 2004;14(6):1188-90.

20. Yang J, Yan R, Roy A, Xu D, Poisson J, Zhang Y. The I-TASSER suite: protein structure and function prediction. Nat Methods. 2015;12(1):7-8.

21. Zheng W, Zhang C, Wuyun Q, Pearce R, Li Y, Zhang Y. LOMETS2: improved meta-threading server for fold-recognition and structure-based function annotation for distant-homology proteins. Nucleic Acids Res. 2019;47(W1): W429-36

22. Maiti R, Van Domselaar GH, Zhang H, Wishart DS. SuperPose: a simple server for sophisticated structural superposition. Nucleic Acids Res. 2004;32:W590-4.

23. Sayle RA, Milner-White EJ. RASMOL: biomolecular graphics for all. Trends Biochem Sci. 1995;20(9):374.
24. Crum RM, Anthony JC, Bassett SS, Folstein MF. Population-based norms for the mini-mental state examination by age and educational level. JAMA. 1993;269(18):2386-91.

25. Nasreddine ZS, Phillips NA, Bedirian V, Charbonneau S, Whitehead V, Collin I, et al. The Montreal cognitive assessment, MoCA: a brief screening tool for mild cognitive impairment. J Am Geriatr Soc. 2005;53(4):695-9.

26. Zhang L, McFarland KN, Jiao J, Jiao Y. A case report of SPG11 mutations in a Chinese ARHSP-TCC family. BMC Neurol. 2016;16:87.

27. Stevanin G, Santorelli FM, Azzedine H, Coutinho P, Chomilier J, Denora PS, et al. Mutations in SPG11, encoding spatacsin, are a major cause of spastic paraplegia with thin corpus callosum. Nat Genet. 2007:39(3):366-72.

28. Salinas S, Proukakis C, Crosby A, Warner TT. Hereditary spastic paraplegia: clinical features and pathogenetic mechanisms. Lancet Neurol. 2008;7(12): 1127-38.

29. Shribman S, Reid E, Crosby AH, Houlden H, Warner TT. Hereditary spastic paraplegia: from diagnosis to emerging therapeutic approaches. Lancet Neurol. 2019:18(12):1136-46.

30. Stevanin G, Azzedine H, Denora P, Boukhris A, Tazir M, Lossos A, et al. Mutations in SPG11 are frequent in autosomal recessive spastic paraplegia with thin corpus callosum, cognitive decline and lower motor neuron degeneration. Brain. 2008;131(Pt 3):772-84.

31. Pan MK, Huang SC, Lo YC, Yang CC, Cheng TW, Yang CC, et al. Microstructural integrity of cerebral fiber tracts in hereditary spastic paraparesis with SPG11 mutation. AJNR Am J Neuroradiol. 2013;34(5):990-6 s991.

32. Winblad B, Palmer K, Kivipelto M, Jelic V, Fratiglioni L, Wahlund LO, et al. Mild cognitive impairment--beyond controversies, towards a consensus: report of the international working group on mild cognitive impairment. J Intern Med. 2004;256(3):240-6.

33. Jacinto-Scudeiro LA, Dariva Machado G, Ayres A, Burguez D, Polese-Bonato M, Gonzalez-Salazar C, et al. Are cognitive changes in hereditary spastic paraplegias restricted to complicated forms? Front Neurol. 2019;10:508.

34. Petersen RC. Clinical practice. Mild cognitive impairment. N Engl J Med. 2011;364(23):2227-34

\section{Publisher's Note}

Springer Nature remains neutral with regard to jurisdictional claims in published maps and institutional affiliations.
Ready to submit your research? Choose BMC and benefit from:

- fast, convenient online submission

- thorough peer review by experienced researchers in your field

- rapid publication on acceptance

- support for research data, including large and complex data types

- gold Open Access which fosters wider collaboration and increased citations

- maximum visibility for your research: over $100 \mathrm{M}$ website views per year

At $\mathrm{BMC}$, research is always in progress.

Learn more biomedcentral.com/submissions 